\title{
Was schützt vor erneuten Exazerbationen?
}

\author{
Das Vermeiden von akuten Exazerbationen gehört zu den Hauptzielen bei der Behandlung von \\ COPD-Patienten. Aber nicht alle Maßnahmen mit nachgewiesener Schutzwirkung sind generell \\ zu empfehlen, und manche sollten trotz fehlenden Nachweises genutzt werden.
}

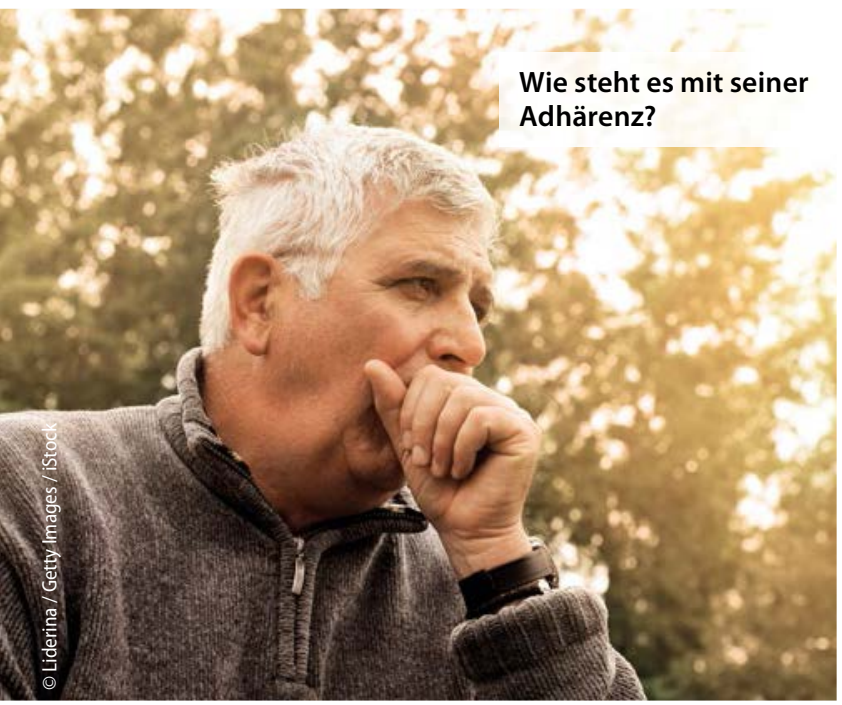

schützt ebenfalls, und zwar besser als Placebo bzw. ICS allein.

5 Patienten in integrierter Versorgung leiden seltener an AECOPD als Patienten mit „Standardversorgung“. Garcia Aymerich empfiehlt deswegen Schulungen für Betroffene.

Wenn die COPD trotzdem exazerbiert, kann durch eine Therapie mit systemischen Kortikosteroiden nicht nur die akute Situation gebessert, sondern auch das Risiko für weitere AECOPD gesenkt werden. Prävention betreiben kann man außerdem, in_ Zur Vorbeugung von akuten Exazerbationen einer COPD (AECOPD) steht ein breites Spektrum an Interventionen zur Verfügung. „Die Prävention geht über die rein medikamentöse Therapie hinaus, und sie ist auch nicht nur Sache des Arztes", betonte Prof. Judith Garcia Aymerich von der Universität in Barcelona bei einem ERS-Symposium.

\section{Fünf wichtige Maßnahmen}

Für Patienten in einer stabilen Phase der Erkrankung haben fünf Maßnahmen eine Schutzwirkung:

1 Die jährliche Influenza-Impfung senkt laut einer Cochrane-Analyse das Risiko für Exazerbationen.

2 Lang wirksame Anticholinergika (LAMA) reduzieren AECOPD und COPD-bedingte Krankenhauseinweisungen.

3 Lang wirksame Betamimetika (LABA) verhindern mittelschwere und schwere AECOPD.

4 Die Kombination aus inhalativem Kortikosteroid (ICS) und LABA dem man Patienten mit einer AECOPD möglichst zu Hause behandelt. Das Konzept „hospital at home“ kommt laut Garcia Aymerich nicht nur dem Wohlbefinden der Patienten zugute, sondern senkt auch das Exazerbationsrisiko. Eine pneumologische Reha nach überstandener AECOPD senkt die Rate weiterer Episoden ebenfalls.

\section{Nutzen-Risiko-Abwägung}

Laut Garcia Aymerich gibt es aber auch vorbeugende Maßnahmen, deren Nutzen nicht die Risiken überwiegt. Das gelte z. B. für die präventive Behandlung mit Makrolidantibiotika. Ein breiter Einsatz komme wegen des Risikos der Resistenzentwicklung und Hörminderung nicht infrage. Bedenken gebe es auch im Hinblick auf die PDE-4-Hemmer - trotz belegter AECOPD-Prävention. So sei z. B. mit einer Zunahme von Insomnien zu rechnen. Für Mukolytika wie N-Acetylcystein haben Studien zwar einen Nutzen angedeutet. Garcia Aymerich beurteilt die Qualität dieser Studien aber als gering. Eine breite Anwendung sei nicht zu empfehlen.
Umgekehrt verhält es sich bei Rauchstopp, Pneumokokkenimpfung und Rehamaßnahmen für stabile COPD-Patienten. $\mathrm{Ob}$ mit diesen Interventionen Exazerbationen verhindert werden, ist bislang nicht ausreichend belegt. Wegen des „herausragenden Nutzens für die COPD-Prognose“, so die Pneumologin, sollte von diesen Maßnahmen aber unbedingt Gebrauch gemacht werden.

Eindeutig nicht zur Exazerbationsprophylaxe zu empfehlen sind laut Garcia Aymerich systemische Kortikosteroide als Dauertherapie sowie Statine.

\section{Gute Adhärenz ist unabdingbar}

Wie gut es gelingt, Exazerbationen zu verhindern, hängt auch von der Mitarbeit des Patienten ab. Er sollte nicht rauchen, körperlich aktiv sein, gesund essen und sich möglichst wenig verschmutzter Luft aussetzen. „Daten zum Nutzen von Bewegung zeigen konsistent ein reduziertes Exazerbationsrisiko“, so Garcia Aymerich. Für besonders kritisch hält sie die Therapieadhärenz - und hier vor allem die korrekte Anwendung der Inhaler. Nach einer Studie aus dem Jahr 2011 werden hier oft Fehler gemacht, weil die Patienten z.B. nach der Beladung in das Mundstück ausatmen, die Inhalation $\mathrm{zu}$ früh beenden oder danach nicht den Atem anhalten.

Die Pneumologin plädierte zudem für Präventionsmaßnahmen auf Populationsebene, wie Rauchverbote und Reduktion von Luftverschmutzung. Nach der Einführung von Rauchverboten in öffentlichen Gebäuden sei ein deutlicher Rückgang von stationären Aufnahmen wegen AECOPD beobachtet worden. -

Dr. Beate Schumacher

- Symposium "The management of acute exacerbations of COPD", Kongress der European Respiratory Society, London, 3.-7. September 2016 